Introduction Hairdressing apprentices are at high risk of developing occupational contact dermatitis.

Materials and methods Data on skin health are presented for 352 hairdressing apprentices attending vocational schools in 24 Croatian towns at the beginning of their education, in a screening phase of a prospective cohort study. Apprentices were recruited from September to December 2017. The study protocol included: Nordic Occupational Skin Questionnaire and International Study on Asthma and Allergy in Children Questionnaire for the evaluation of self-reported skin and atopy symptoms, clinical skin examination interpreted by means of Osnabrueck Hand Eczema Severity Index (OHSI), genotyping filaggrin (FLG) gene polymorphisms 2282del4 and R501X from buccal swabs, skin $\mathrm{pH}$ and transepidermal water loss (TEWL) measurements.

Results In the total sample $(n=352$, median age 15,18 males), a history of respiratory and/or skin atopy symptoms was reported by $44.89 \%$, hand/wrist eczema by $11.93 \%$, and a history of dry hands (without eczema) by $34.38 \%$ of apprentices. One or more hand/wrist skin changes were found at the clinical examination in $18.18 \%$ of apprentices, with the OHSI score ranging from 1 to 6 . Washing hands $>20$ times per day was identified in $12.78 \%$ of apprentices. An FLG gene mutation (R501X) was found in only one apprentice. The median (range) for hand TEWL and $\mathrm{pH}$ was 13.1 (4.36-62.69) and 5.68 (4.28-7.13), respectively. OHSI score was positively correlated with hand TEWL (Spearman rho 0.16; $\mathrm{p}=0.0026$ ), and $\mathrm{pH}$ (Spearman rho $0.13 ; \mathrm{p}=0.0186)$.

Conclusion The results indicate a high prevalence of selfreported atopy $(45 \%)$ and moderate prevalence of selfreported (12\%) and clinically observed skin symptoms (18\%) on the hands/wrists of hairdressing apprentices already at the beginning of education, without FLG mutations as a risk factors. This emphasizes the need to ameliorate preventive examinations of children before enrolling to schools for professions with high risk of exposure to skin hazards.

\section{B.4 WORKERS' COMPENSATION CLAIMS FOR OCCUPATIONAL CONTACT DERMATITIS: 20 YEARS OF DATA FROM VICTORIA, AUSTRALIA}

\footnotetext{
1,2Tessa Keegel*, 1,20llie Black, ${ }^{*}$ Ewan MacFarlane, 1,2Rwth Stuckey, ${ }^{2,3}$ Anthony LaMontagne, ${ }^{4}$ Rosemary Nixon, ${ }^{2}$ Malcolm Sim. ${ }^{1}$ Department of Public Health, La Trobe University, Melbourne, Australia; ${ }^{2}$ Monash Centre for Occupational and Environmental Health, Monash University, Melbourne, Australia; ${ }^{3}$ Centre for Population Health Research, Deakin University, Melbourne, Australia; ${ }^{4}$ Skin and Cancer Foundation, Melbourne, Australia
}

\subsection{6/OEM-2019-EPI.119}

Background Occupational contact dermatitis is one of the most common occupational diseases, but there is a lack of reliable information on incidence. Despite acknowledged limitations, workers' compensation statistics may provide insights into contact dermatitis patterns.

Objective The objective of the study was to characterise historical patterns of workers' compensation claims for occupational contact dermatitis.

Methods This was a retrospective analysis of workers' compensation claims for occupational contact dermatitis from 1996-2015 $(n=3,348)$ accepted by WorkSafe Victoria in Victoria, Australia. Accepted claims per 100000 person-years stratified by sex, age and industry were calculated.
Denominators for the population at risk were obtained from the Australian Bureau of Statistics using Victorian Labour Force Survey data.

Results The compensation claims rate of occupational contact dermatitis was 6.72 per 100000 person-years for the overall twenty-year period. There was a significant reduction in claims from 11.84 in 1996 to 1.78 in 2015. Males had a higher overall claims rate of 7.97 compared to the rate for females of 5.18. Over the twenty-year period the rate for males decreased from 14.46 to 1.7 compared to a reduction from 8.4 to 1.8 for females. This decrease was still observed when the data were standardised for underlying changes in the age structure of the population. There was an overall decline across all high-risk occupational groups

Conclusions There was a fivefold decrease in accepted claims for occupational contact dermatitis for the twenty-year period from January 1996 to December 2015 for the state of Victoria in Australia. These results need to be regarded with caution as the declining rate of accepted occupational contact dermatitis claims may indicate changes in workplace dermal exposures or improvements in workplace skin protection practices over time, or they may be driven by underlying changes to the workers' compensation system or changes to claims behaviour amongst workers.

\section{B.5 EFFECTIVENESS OF A SKIN CARE PROGRAM FOR THE PREVENTION OF OCCUPATIONAL CONTACT DERMATITIS IN HEALTHCARE WORKERS}

Henk van der Molen*, Sanja Kezic*, Maryam Soltanipoor, Thomas Rustemeyer. Amsterdam UMC, Amsterdam, Netherlands

\subsection{6/OEM-2019-EPI.120}

Healthcare workers (HCW) are at risk for developing hand dermatitis (HD) caused by exposure to wet work. Guidelines for the prevention of HD recommend regular use of moisturizers, however in practice their use remains low and their effectiveness is poorly investigated.

The main objective of this randomized control trial was to assess whether an intervention aimed at improving skin care leads to reduction in HD severity. The intervention included provision of cream dispensers with electronic monitoring of use, regularly communicated to the HCW. The primary and secondary outcomes were change from baseline in Hand Eczema Severity Index score ( $\square$ HECSI) and Natural Moisturizing Factor ( $\square \mathrm{NMF}$ ) levels as a biomarker of early changes in the skin barrier. Nine wards $(285 \mathrm{HCW})$ were allocated to an intervention group (IG) and 10 wards $(216 \mathrm{HCW})$ to the control group (CG).

At baseline, IG and CG had similar exposure to wet work, use of skin care and severity of HD. At follow-up (1 year) the IG showed significantly higher frequency of hand cream use as compared to the CG (self-reported data). Though, electronically collected data in the IG showed that the average frequency of 0.4 cream applications/shift was far below recommended 2 applications/shift. The HECSI reduced significantly in the IG for $-6.2(95 \% \mathrm{CI}-7.7,-4.7)$ and in the CG -4.2 points $(95 \% \mathrm{CI}-6.0,-2.4)$. There was no difference in $\square$ HECSI or $\square$ NMF between IG and CG, however the subgroup showing mild symptoms showed significantly larger improvement in HD symptoms as compared with CG. 
The intervention proved overall positive effects on severity of HD symptoms, supporting the benefits of skin care in the workplace. The fact that the intervention showed a larger effect in a subgroup with mild symptoms suggests that this intervention might be of particular importance for primary prevention.

\section{B.6 OCCUPATIONAL CONTACT DERMATITIS AND HOW THE COMMUNITY RESPONSES IN DEVELOPING COUNTRY}

Shambhu Joshi*. Far Western Community Hospital, Kailali, Nepal

\subsection{6/OEM-2019-EPI.121}

Background Dermatologic disorders generally have a major impact on patients daily activities, psychological and emotional state and social relationships in a resource poor setting. Country with different climate zones and environment as well as traditional use of herbal and increasing use of pesticides contact dermatitis is one of the important occupational hazards in construction workers, nearby people and it often leads to poor quality of life of the workers with substantial financial loss.

Methods This study was conducted in three different geographic population. Free health camp was organized with total 310 cases of dermatologic problems were registered in Skin OPD last two years. Patients includes children to geriatric population both male and female groups.

Results Out of the total $42 \%$ were male and $58 \%$ were female patients. Contact dermatitis were found among 28\% of the patients, age and sex had no significant role on prevalence of Contactdermatitis. Urban area patients have tendency due to hair dye, industrial cement and detergents, however rural area patients have causation due to herbal plants (Parthenium dermatitis) and traditional occupational works. Both have nickel, atopic and unknown cause. Foot was the most commonly involved site $(25.7 \%$ of patients). Atopy was present in 18 patients $(25.7 \%)$. A total of 22 irritant reactions were noted in 13 patients. Most of the patients neglect the contact dermatitis due to lack of health information and use of irrational medication also seen.

Conclusion Most of the contact dermatitis is preventative and manageable in time. Due to Climate, geographical areas, literacy difference community preventive health program plays important role, it. Prick-Patch testing, Teledermatology and Geographical information system is another future perspective for dermatology disease mapping in Nepal so that the government can make essential policy, programs for communities so that we can prevent and treat the contact-dermatitis on time.

\section{Mini-Symposium 3: Military Epidemiology}

\section{C.1 THE NZ VIETNAM VETERAN FAMILY STUDY: A MULTI- GENERATIONAL PERSPECTIVE ON HEALTH AND WELLBEING}

David McBride*, Amy Richardson, Brian Cox. University of Otage, Dunedin, New Zealand

\subsection{6/OEM-2019-EPI.122}

Background The serious impact of Vietnam service on New Zealand veterans arose from high levels of combat experience and exposure to chemical agents including Agent Orange. In 2009, we assembled a cohort of 2783 men and 23 women Vietnam veterans. They had significantly increased risks of head and neck cancer and chronic lymphatic leukaemia, with high rates of hospitalisation for renal failure and drug and alcohol problems. Overseas data indicates that families have been affected by relationship difficulties, with subsequent risks of adverse psychological outcomes in children. There have been few longitudinal studies which include a comprehensive exposure assessment (the exposome) and include both veteran and family, which is what we propose.

Study population Using the cohort data, the electoral roll and an information campaign, we will recruit veterans, their spouses and their children for follow up. For all groups, we will obtain data to inform the general exposome through a base-line health survey using some of the brief measures deployed in our 'contemporary veterans' study, including the Post Traumatic Checklist (Civilian or military) for PTSD, and the Brief Family Relationship Scale exposure history.

The specific exposome, including chemicals and 'other' specific exposures, will be recorded by on-line questionnaire and subsequent interview.

Effects will be assessed through linkage to the Birth Defects Register, Mortality Collection, routine datasets for hospital discharge, and the Cancer Registry, using comparisons with national rates where appropriate. Most importantly, permission will be sought to flag individuals at the Cancer Registry, with access to blood or tissue samples facilitating the identification of genomic 'effect biomarkers'. We hope that the method will be extended to other cohorts and for future deployments.

\section{C.2 LONG TERM PHYSICAL AND MENTAL HEALTH IMPACT OF MILITARY SERVICE}

Malcolm Sim*, Jing Xie, Andrew Forbes, Helen Kelsall. School of Public Health and Preventive Medicine, Monash University, Melbourne, Australia

\subsection{6/OEM-2019-EPI.123}

Background There is emerging recognition of the important co-morbidities and long term relationships between physical and psychological health in military and veteran populations. The aim was to investigate the longitudinal relationships between multisymptom illness (MSI) and psychological disorders.

Methods A cohort of 1990-1991 Gulf War veterans and military comparison group was assessed at Wave 1 (2000-2002) and Wave 2 (2011-2012), including military service characteristics, symptoms, modified Centers for Disease Control (CDC) definition of MSI, the posttraumatic stress disorder (PTSD) Checklist (PCL), and Alcohol Use Disorder Identification Test (AUDIT). The Composite International Diagnostic Interview (CIDI v.2.1) assessed psychological disorders using DSM-IV criteria. Incident cases were defined as participants who did not meet criteria for a health outcome at Wave 1 but met these criteria at Wave 2.

Results Overall participation at Wave 2 was 1390/2779 (50.0\%); 99.8\% completed a health questionnaire including 1356 male participants who were included in these analyses. The adjusted incident rate ratio (IRR) of MSI at Wave 2 was higher for those with, compared to those without, CIDI- 